\title{
Effect of Convective, Diffusive and Source Terms in Self Generated Magnetic Field due to Laser Plasma Interactions
}

\author{
S. Khanal and R. Khanal \\ Central Department of Physics, Tribhuvan University, Kirtipur, Kathmandu, Nepal
}

\begin{abstract}
Laser-plasma interaction phenomenon has various applications and the most important one is in the Inertial confinement Fusion. Spatial and temporal variations of self generated magnetic field have been studied within the framework of magnetohydrodynamics. The evolution equation that describes the generation of magnetic field is solved using complex Fourier and Laplace transformation methods as an initial value problem. Convective, diffusive and source terms are considered in the evolution equation and are solved theoretically. Magnetic fields of the order of megagauss have been obtained among which the maximum field is just about $40 \mathrm{MG}$. The results are comparable with earlier reported results.
\end{abstract}

Keywords: plasma, inertial cofinement fusion, self generated magnetic field, magnetohydrodynamics

\section{INTRODUCTION}

Self-generated magnetic field in laser plasma interactions were firstly found in gas breakdown which was carried out by Korobkin and Serov in 1966 [1]. Afterwards, its study had been conducted with great interest by various scientists in many laboratories experimentally as well as theoretically. Stamper et al. recorded the first measurement of self generated magnetic field in laser produced plasma and they explained it on the basis of thermal source [2]. The interaction between intense laser radiation and plasma is a very important phenomenon in coronal plasma physics. Due to the absorption of laser in plasma it creates suprathermal electrons and electrostatic plasma which results in the steepening of the density profile. It is found that various non linear effects are produced during these types of interactions such as fast electron and ion generations producing strong electric as well as magnetic fields. Various experiments performed on laser plasma interactions showed that strong magnetic fields of the order of megagauss are generated during these interactions. Extremely high magnetic fields of the order of few megagauss play an important role in energy transport, propagation of laser pulses, laser beam self focusing, penetration of laser radiation into the overdense plasma and plasma electron and ion acceleration. Furthermore, such types of self generated magnetic fields have great concern for the target designers to conduct laser fusion because these fields may affect in the inhibition of transport of thermal energy into the target. The effect of self generated magnetic field in energy transport, energy deposition showed that it play vital role in inertial confinement fusion.

Because of these reasons the study of self generated megagauss magnetic field is still a topic of interest. Recently, Gopal et al. [3] has reported the experimental studies on megagauss magnetic field generation using a 35 femtosecond laser at relativistic intensities. Series of papers [4]-[11] had been published on the study of self generated megagauss magnetic field which has made an important improvement on the progress of design of inertial confinement fusion.

\section{MODEL}

The equation that governs the magnetic field generation in conducting fluid is solved by using the magnetohydrodynamic approach also called MHD. Starting with the momentum balance equation for electrons we have derived the Generalized Ohm's law and finally the evolution equation as [12],

$$
\begin{aligned}
\frac{\partial \vec{B}}{\partial t}=\nabla \times \vec{V} \times \vec{B} & -\frac{\eta}{\mu_{0}} \nabla \times(\nabla \times \vec{B})+\frac{1}{\mu_{\mu^{\prime}} e} \nabla \times \frac{1}{n_{0}}\left[\nabla\left(\frac{B^{2}}{2}\right)-(\vec{B} . \nabla) \vec{B}\right]+\frac{1}{n_{e} e} \nabla \\
& \times\left(\nabla p_{t}+\nabla p_{r}\right)-\frac{m_{e}}{e^{2} n_{0}} \nabla \times \vec{j}-\frac{1}{e} \nabla \times\left(\beta \nabla K T+\frac{1}{e} \alpha \hat{b} \times \nabla K T\right)
\end{aligned}
$$

where $\vec{V}, e, n_{0}$ and $T$ are velocity, number density, charge and temperature of the electron respectively. 
$\eta$ and $\mu_{0}$ are plasma resistivity and magnetic permeability of plasma respectively. $\alpha$ and $\beta$ are the thermal transport coefficients. $J$ is current density and $\mathrm{K}$ is the Boltzmann constant.

The evolution equation (1) is highly nonlinear in nature and contains terms like convective, diffusive, source, Hall, thermal force, radiation pressure etc. Among these terms source term, diffusive term and convective term have dominant effect whereas remaining terms have negligible effect on the self generated magnetic field.

We have taken three dominant terms namely convective, diffusive and source terms two at a time. In the first model we have taken the convective term and thermal source term and solved analytically using initial value problem to obtain the evolution of magnetic field as,

$$
\vec{B}(z, t)=\frac{K T}{e L_{n} L_{T}} \frac{\Delta n}{n} \exp \left(-\frac{Z}{L_{n}}\right)\left[1-\exp \left(-\frac{t}{\tau}\right)\right] \tau
$$

where, $\mathrm{t}$ and $\tau$ are called evolution time and characteristic time respectively. $L_{n}$ and $L_{T}$ are density scale length and temperature scale length respectively whereas $\mathrm{z}$ is the axial length.

In the second model we have taken the diffusive term and the source term. The source term is taken as [13],

$S(\vec{r}, t)=S_{t}(\vec{r}, t)+S_{r}(\vec{r}, t)=\frac{1}{e} \nabla \times \frac{1}{n_{0}} \nabla p_{t}+\frac{1}{e} \nabla \times \frac{1}{n_{0}} \nabla p_{r}$

In this equation $S_{t}(\vec{r}, t)$ and $S_{r}(\vec{r}, t)$ are the thermoelectric source and radiative source terms respectively. By solving the evolution equation taking diffusive and source terms only from the evolution equation we finally obtained the magnetic field as equation (3).

$$
\vec{B}(\vec{r}, t)=S_{0} \int_{0}^{t} f\left(\omega_{0} \tau\right) d \tau e^{-\frac{a r^{2}}{\left[1+\frac{4 n a(t-\tau)}{\mu_{0}}\right]}}\left[1+\frac{4 \eta a(t-\tau)]^{-3 / 2}}{n}\right.
$$

where, $\mathrm{S}_{0}$ is source strength whose value has been taken equal to that of thermal source. Its dimension is magnetic field per unit time. $f\left(\omega_{0} \tau\right)$ is the time part of the source with $f\left(\omega_{0} \tau\right)$ being characteristic oscillation frequency of the magnetic field source. is a constant that represents the square of the reciprocal skin depth. In our work skin depth is represented by $R_{0}$.

\section{RESULTS AND DISCUSSION}

The evolution equation that represents self generated magnetic field contains various terms. All terms of this equation are not equally important. Among these terms convective, diffusive and source terms have dominant effect on self generated magnetic field while the remaining terms have negligible effect. In our work, firstly we have taken convective term and thermal source term and obtained the evolution of magnetic field as equation (2). The temporal and spatial variation has been seen with magnetic field by taking the following values of the parameters which are consistent with the earlier papers [4] [5] [8] [14 - 16]. $T=(1$ to 5$) \mathrm{KeV}, L_{n}=35 \mu \mathrm{m}, L_{T}=1 \mu \mathrm{m}, \mathrm{z}=1 \mu \mathrm{m}, \tau=100 \mathrm{ps}$.

Secondly, we have taken the following values of the parameters which are again consistent with the earlier reported values.

$$
\begin{aligned}
& L_{n}=1 \mu m, L_{T}=1 \mu m, t=100 n s, T_{0}=6 n s, \\
& R_{0}=100 \mu m, a=\frac{1}{R_{0}^{2}}, \omega_{0}=\frac{2 \pi}{T_{0}}, T_{e}=300 \mathrm{KeV}
\end{aligned}
$$

The Coulomb logarithm and plasma resistivity $(\eta)$ were calculated using the formulae [2],

$$
\Lambda=12 \pi n^{-1 / 2}\left(\frac{\varepsilon_{0} K T_{e}}{e^{2}}\right)^{3 / 2} \quad \eta=\frac{\pi e^{2} m_{e}{ }^{1 / 2}}{\left(4 \pi \varepsilon_{0}\right)^{2}\left(K T_{e}\right)^{3 / 2}} Z \ln \Lambda,
$$

$\mathrm{Z}$ is the ionic charge number of plasma target. The results of our work have been summarized in the following figures.

We have studied the effect of convective, diffusive and source terms in self-generated magnetic field due to laser plasma interactions based on the evolution equation of magnetic field as suggested by Braginskii [17]. By using the evolution equation, temporal and spatial variation of magnetic field have been presented by taking different parameters. The values of the parameters taken are consistent with previously reported values. The magnetic field has 
been calculated by considering convective, diffusive and source terms taking two at a time.

Firstly we have taken thermal source term and convective term and studied the spatial and temporal variation of magnetic field. Secondly we have taken the thermal source term, radiative source term and diffusive term which are used to study the spatial variation of magnetic field. When taking convective and thermal source terms, the evolution equation has been solved using initial value problem by adopting Laplace transformation method. Figures 1, 2 and 3 are plotted by using equation (2). Fig. 1 and Fig. 2 show the variation of magnetic field with $t / \tau$ for different values of electron temperatures and different values of ${ }^{Z} / L_{n}$ respectively. Both the curves show the field saturation with increasing time. This saturating behavior is in good agreement with previous works [4] [9]. In both curves of Fig. 1 and Fig. 2 the saturation values of magnetic field for $1 \mathrm{KeV}$ temperature are obtained to be $7.5 \mathrm{MG}$ and 2.8 MG. Fig. 3 shows the variation of magnetic field with ${ }^{Z} / L_{n}$ for varying $t / \tau$. The peak value of magnetic field is obtained to be $3.1 \mathrm{MG}$ for $t / \tau=0.5$. The curve shows that the peak value of magnetic field is maximum for lower value of $z / L_{n}$ and higher value of $t / \tau$. Also magnetic field is found to decrease gradually on increasing $z / L_{n}$ and finally it tends to zero. It shows that the self generated magnetic field produced in laser plasma interactions is of short range.

While considering the diffusive and source terms (thermal as well as radiative source), we solved the evolution equation using initial value problem by adopting Fourier and inverse Fourier transformation in space and Laplace and inverse Laplace transformation in time. In this case we have taken the source term in the form of oscillating Gaussian profile $f\left(\omega_{0} t\right)=\sin \omega_{0} t$ : And the source amplitude is taken to be that of the thermoelectric source $S_{0}=\frac{K_{B} T_{e}}{e L_{n} L_{T}}$.

Fig. 4 and Fig. 5 show the spatial variation of magnetic field for different ionic charge number of plasma targets and number densities respectively. It shows the decay in magnetic field with increasing values of $r / R_{0}$. The maximum value of magnetic field is obtained for lower ionic charge number and higher densities. The maximum obtained magnitude of the magnetic field is just about $40 \mathrm{MG}$.

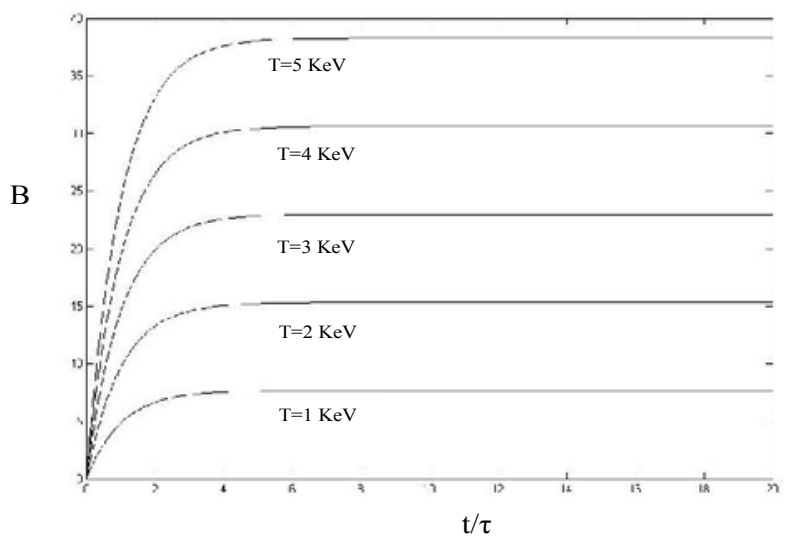

Fig. 1: Variation of the magnetic field with $t / \tau$ for different electron temperatures

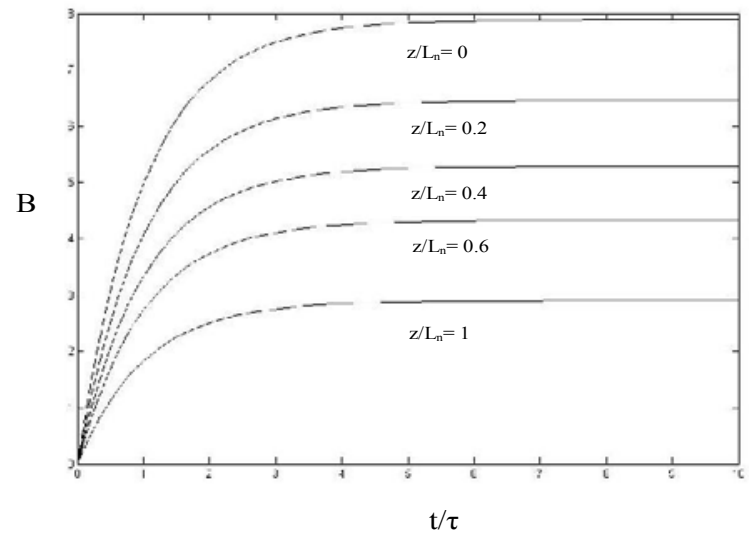

Fig. 2: Variation of the magnetic field with $t / \tau$ for different values of $\mathrm{z} / \mathrm{Ln}$

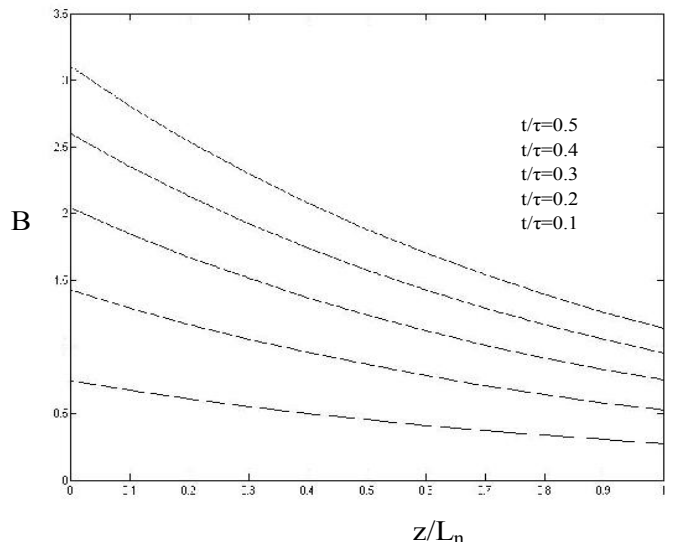

Fig. 3: Variation of the magnetic field with $\mathrm{z} / \mathrm{ln}$ for different values of $\mathrm{t} / \tau$ 


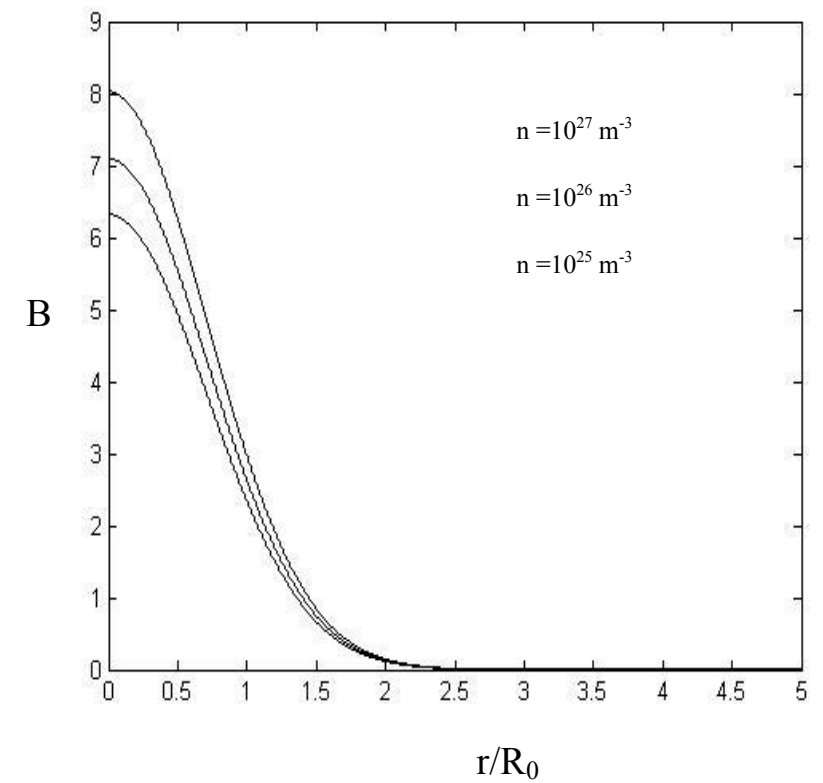

Fig. 4: Variation of magnetic field with $\mathrm{r} / \mathrm{R} 0$ for different number densities

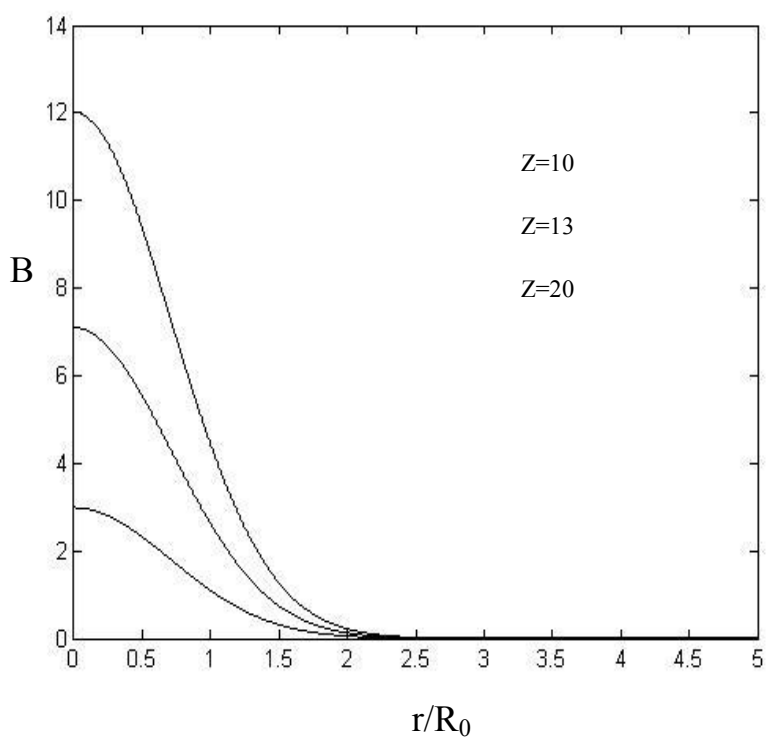

Fig. 5: Variation of magnetic field with $\mathrm{r} / \mathrm{R} 0$ for different ionic charge number $Z$

\section{REFERENCES:}

[1] V. V. Korobkin and R. V. Serov, JETP Lett. 4, 70 (1966)

[2] J. A. Stamper et al., Phys. Rev. Lett. 26, 1012 (1971)

[3] A. Gopal et al., Plasma Phys. Control Fusion 55, 035002(2013)

[4] M. S. Bawa'aneh, IEEE Transactions on Plasma Science, 38, 1808 (2010)

[5] C. A. Cecchetti et al., Phys. Plasmas 16, 043102 (2009)

[6] E. L. Clark et al., Physical Review Lett. 84, 670 (2000)

[7] A. J. Kemp, B. I. Cohen and L. Divol, Livermore National Laboratory, Internal report (2009)

[8] L. Willingale et al., Plasma Phys. Control Fusion 105, 095001 (2010)

[9] A. Gopal et al., Phys. Plasmas 15, 122701 (2008)

[10] H.-S. Park et al., Physical Review Lett. 112, 055001 (2014)

[11] S. J. Rose, Physics 7, 13 (2014)

[12] S. Khanal, Effect of convective, diffusive and source terms in self generated magnetic field due to laser plasma interactions, M.Sc. Thesis, Tribhuvan University, Kirtipur, Nepal (2012)

[13] P. Mora and R. Pellat, Phys. Fluids, 24, 2219 (1981)

[14] M. Tatarakis et al., Phys. Plasmas 9, 2244 (2002)

[15] M. Tatarakis et al., Central Laser Facility, CLRC Rutherford Appleton Laboratory UK, Annual report $(2001 / 2002)$

[16] J. J. Nakarmi and L. N. Jha, International Centre for Th. Physics, Trieste, Italy:- Internal Report IC/96/259 (1996)

[17] S. I. Braginskii and M. A. Leontovich, Reviews of Plasma Physics, 1, 205 (1965) 